\title{
Cynicism as subscale of burnout
}

\author{
Viljoen M; Claassen N
}

\section{Abstract}

Background: Cynicism, as a personality trait, has adverse effects on health. The question was asked whether cynical attitudes that develop due to work-related stress correlate with stress levels and whether it has a negative influence on health.

Objectives: To investigate associations of the cynicism subscale scores of the Maslach Burnout Inventory-General Survey (MBI-GS) with levels of stress, anxiety, questionnaire-based physical health and with a number of physiological health risk indicators.

Methods: Cynicism, anxiety, questionnaire-based physical health scores, as well as allostatic load, heart rate variability and C-reactive protein, were assessed in 27 males working between 40 and 80 irregular hours per week.

Results: Cynicism scores related to stress levels $(r=0.411, p=0.030)$. Effects of work-related cynicism on physical health were suggested by negative associations with questionnaire-based scores on physical health $(r=-0.383, p=0.044)$ and heart rate variability indicators $(r=-0.379, p=0.047$ to $r=-$ $0.496, p=0.007)$, and by positive associations with anxiety levels $(r=0.408, p=0.031)$, heart rate $(r=0.449, p=0.017), B M I(r=0.426, p=0.024)$ allostatic load $(r=0.360, p=0.065)$ and levels of the inflammatory marker C-reactive protein $(r=0.407, p=0.035)$.

Conclusions: Cynicism, as reflected by the MBI-GS, increases with increases in stress levels and could contribute to the decline in the health reported for burnout.

Keywords: Cynicism, stress, health, workplace. 


\section{Introduction}

Burnout is a work-related stress syndrome characterized by vital exhaustion, a cynical attitude towards work and a tendency to evaluate oneself negatively with regard to work efficacy [1]. Burnout has been linked to disturbances such as impaired attention and memory, incident dementia, sleep disturbances, depression, anxiety, decreased work capacity, sickness absence from work and a host of poor health behaviours $[2,3,4]$. It has further been implicated in somatic syndromes such as cardiovascular diseases, and evidence is emerging for associations between burnout and disorders such as the metabolic syndrome, systemic inflammation, and dysregulation of coagulation functions $[2,5]$. Although some inconsistencies exists about the psychophysiological consequences of burnout and the scales to be included in the assessment tools, one view that seems to be generally agreed upon is that burnout represents a condition of chronic stress, and that the energy-depleted state of exhaustion embodies the primary characteristic of burnout [1,2].

Several validated burnout measuring instruments exist, most comprising of an exhaustion and two other subscales. The Maslach Burnout Inventory-General Survey (MBI-GS), for instance, assesses burnout in terms of three subscales, i.e., exhaustion, cynicism and professional efficacy, where cynicism is said to reflect an indifferent or distant attitude toward one's work [1]. In contrast, some researchers have argued for an "exhaustion only" model of burnout and such assessment models have indeed been developed $[2,6]$.

Many attempts have been made to find physiological correlates or biomarkers for burnout, but absolute consensus has not yet been reached [7]. Nevertheless, substantial evidence exists that stress-induced dysregulations of the main stress axes, i.e., the hypothalamo-pituitary-adrenocortical axis (HPA-axis) and the autonomic nervous system (ANS), are present in burnout $[2,5,7,8]$. Unfortunately results, as for many other stress-related disorders, are confounded by assessments at different stages and degrees of burnout. For example, while chronic stress initially results in hypercortisolemia, it may eventually lead to hypocortisolemia [9] - a fact that can, and have indeed, 
lead to opposite findings in the search for biological correlates of burnout. At present there is enough evidence to accept deficiencies in HPA axis and ANS to be biological correlates of severe burnout $[8,10,11]$. In view of the role of the HPA axis (cortisol) and the ANS in energy availability, coupled to associations of hypocortisolism and ANS insufficiency with fatigue $[8,9])$, it seems feasible to accept inadequacies in these main stress axes $[8,10,11]$ as correlates of the burnout-associated exhaustion. In addition, due to the loss of glucocorticoid regulation of the immune system, hypocortisolaemia can lead to an increase in pro-inflammatory cytokines [12]. An increase in proinflammatory cytokines is, in turn, associated with a range of pathophysiological and psychopathological disturbances marked by chronic fatigue [13]. It is of interest to note the many disturbances for which an association have been reported between stress, fatigue, inflammatory cytokines and dysregulation of the HPA-axis, e.g., severe burnout, chronic fatigue syndrome, fibromyalgia, atypical depression, and sickness behaviour $(12,14,15,16,17,18]$.

With most of the biological correlates described for burnout [7] directly or indirectly relating to exhaustion, it is tempting to agree with the "exhaustion/fatigue only" view [6] of what burnout constitutes. However, burnout is by definition not a context free phenomenon, but job stress-related and marked by emotional disengagement from (cynicism towards) the work situation and feelings of reduced professional efficacy $[1,19]$. As cynicism as a personality trait is known to negatively affects wellbeing it was of interest to us to see whether the cynicism that develops in the face of work-related stress is also associated with a decline in health.

We were interested in cynicism as subscale of burnout, and specifically cynicism as assessed as by the subscale of the Maslach Burnout Inventory-General Survey (MBI-GS) [1]. This writing reports on associations of the MBI-GS cynicism subscale scores with stress levels and with a number of health risk indicators, including allostatic load, heart rate variability (HRV), C-reactive protein (CRP), as well as questionnaire-based assessments of stress, anxiety levels and physical health. 


\section{Methods}

Burnout was assessed by the MBI-GS [1]. Stress levels were estimated by the Experience of Work and Life Circumstances Questionnaire [20], anxiety by the State-Trait Anxiety Inventory for Adults [21 and a summary score on physical health by the SF-36v2 Health Survey [22].

Allostatic load, a pathophysiological index of the accumulated effects of stress, is considered an indicator of risk for decline in cognitive and physical functioning, including cardiovascular disease [23]. Allostatic load was measured by an adaptation of the method of Seeman, et al [23]. Biomarkers used for assessment of allostatic load were systolic and diastolic blood pressure, waist-to-hip ratio, body mass index (BMI), serum HDL, serum LDL, total cholesterol, aldosterone, serum albumin, glycosylated haemoglobin, overnight urinary cortisol excretion, fasting blood glucose and serum CRP. CRP is an acute phase protein, used as biomarker of pro-inflammatory activity. Elevated proinflammatory activity, as reflected by CRP levels, has been described in association with stress and life style associated diseases [24]. HRV reflects autonomic nervous system input to the heart and a decrease in HRV has been reported for cardiovascular and non-cardiovascular diseases [25]. Tachogram recordings for the determination of HRV were performed under controlled laboratory conditions and analysed in 5 min periods. HRV analysis was performed in line with recommendations by the HRV Task Force [25], using the advanced HRV Analysis 1.1 software for windows. Error correction was performed with the Polar Precision Performance Software version 4.03 .040 and detrending by the smoothness priors method [26]. Total HRV, high frequency power (HF) and low frequency power (LF), as obtained by non-parametric frequency-domain analysis based on Fast Fourier Transformation [25], are presented. HF reflects parasympathic and LF is said to be a combination of sympathetic and parasympathetic control of cardiac function.

Twenty-seven volunteers signed informed consent prior to inclusion in the study. All subjects were fulltime employed males and all worked irregular hours ranging from 40 to $80 \mathrm{hrs}$ per week. Clearance was received from the Ethics Committee, Faculty of Health Sciences, University of Pretoria 
and the study was thus performed in accordance with the ethical standards laid down by the Declaration of Helsinki.

\section{Results}

MB-GS scores were $4.5 \pm 4.2$ for exhaustion; $6.6 \pm 4.3$ for cynicism and $31.9 \pm 4.4$ for professional efficacy. No correlation of statistical significance was found between scores on the exhaustion subscale and that on the cynicism subscale, or between scores on the cynicism subscale and that on professional efficacy. Not statistically significant, but pointing towards a trend was the inverse correlation between the exhaustion subscale and that for professional efficacy $(r=-0.329, p=0.088$. The mean allostatic load was 4.4.土2.4. Correlations between scores for MBI-GS subscales and other health indicators are shown in Table 1. Associations where at least one of the three subscales showed significant correlations $(p<0.05$,$) or correlations indicating a trend (0.05<p \leq 0.100)$ are presented.

4. Table 1: Correlations between MBI-GS subscales and health risk indicators.

\begin{tabular}{lcccccc}
\hline Potential risk & \multicolumn{2}{c}{ MBI Exhaustion } & \multicolumn{2}{c}{ MBI Cynicism } & \multicolumn{2}{c}{ Professional efficacy } \\
\cline { 2 - 7 } indicator & $\mathrm{r}$ & $\mathrm{p}$ & $\mathrm{r}$ & $\mathrm{P}$ & $\mathrm{r}$ & $\mathrm{p}$ \\
\hline Age & 0.179 & 0.363 & $0.401^{*}$ & 0.034 & -0.164 & 0.406 \\
Allostatic load & 0.316 & 0.108 & 0.360 & 0.065 & -0.348 & 0.075 \\
Stress levels & $0.447^{*}$ & 0.017 & $0.411^{*}$ & 0.030 & -0.060 & 0.760 \\
Trait anxiety & 0.159 & 0.418 & $0.408^{*}$ & 0.031 & -0.132 & 0.503 \\
Phys health & $-0.429^{*}$ & 0.023 & $-0.383^{*}$ & 0.044 & 0.341 & 0.076 \\
BMI & $0.409^{*}$ & 0.031 & $0.426^{*}$ & 0.024 & -0.287 & 0.139 \\
CRP & $0.417^{*}$ & 0.030 & $0.407^{*}$ & 0.035 & -0.150 & 0.456 \\
HR & 0.098 & 0.621 & $0.449^{*}$ & 0.017 & -0.238 & 0.223 \\
BP $_{\text {Standing }}$ & 0.327 & 0.089 & 0.283 & 0.145 & -0.327 & 0.090 \\
Total HRV $_{\text {Supine }}$ & -0.374 & 0.050 & $-0.379^{*}$ & 0.047 & 0.238 & 0.222 \\
Total HRV $_{\text {Standing }}$ & -0.224 & 0.252 & $-0.416^{*}$ & 0.028 & 0.334 & 0.082 \\
LF Supine $_{\text {SF }}$ & $-0.415^{*}$ & 0.028 & $-0.398^{*}$ & 0.036 & 0.263 & 0.177 \\
LF $_{\text {Standing }}$ & -0.263 & 0.177 & $-0.496^{* *}$ & 0.007 & $0.390^{*}$ & 0.040 \\
HF $_{\text {Supine }}$ & -0.238 & 0.222 & -0.319 & 0.098 & 0.184 & 0.350 \\
HF $_{\text {Standing }}$ & -0.117 & 0.555 & -0.327 & 0.089 & 0.313 & 0.105 \\
\hline
\end{tabular}

5. Calculation of Spearman correlations were performed with SPSS, version 23. Significant correlations $(p<0.05)$ and correlations indicating a trend $(0.05<p \leq 0.100)$ are shown. $B M I=$ body mass index, $\mathrm{CRP}=\mathrm{C}$-reactive protein, $\mathrm{HR}$ =heart rate, $\mathrm{BP}_{\text {syst }}=$ systolic blood pressure, $\mathrm{HRV}=$ heart rate variability, $\mathrm{HF}=$ high frequency, $\mathrm{LF}=$ low frequency, Phys health= summary score on questionnaire-based health assessment with higher scores denoting better health. 


\section{Discussion}

Burnout is a work-related stress syndrome with negative consequences for physical and mental health. Various burnout measuring scales exist, which, but for exhaustion, differ in subscales characteristics. General agreement exists on exhaustion as primary characteristic of burnout, and credible indications exist for physiological correlates that could explain the pathophysiological symptoms of this energy-depleted state of exhaustion $[10,11]$. However, the same cannot necessarily be said for other characteristics included as subscales. This pilot study investigated associations between the cynicism subscale scores of the MBI-GS and a number of stress-related health risk indicators.

Cynicism as a personality trait is implicated as a contributing factor to cardiovascular and immunological disorders, all-cause mortality, and mental disturbances such as incident dementia $[27,28,29,30,31]$. However, cynicism as a personality trait and cynicism that develops as a result of unfavourable work condition differ. Cynicism towards the work situation, as assessed in burnout inventories, is equated to disengagement from work as a result of work-related stress and although cynicism as a personality trait could very well have an impact, it is not necessarily a prerequisite for becoming cynical about the workplace.

In the present study, against expectations, correlations with statistical significance were more often seen with cynicism than with exhaustion (Table 1). Cynicism was positively associated with stress, confirming the increase in cynicism with increases in stress levels, as postulated by the MBI-GS and other Maslach Burnout Inventories [1].

A positive correlation was also found between cynicism and trait anxiety, but not state anxiety. This association with trait, but not state anxiety appears to suggest that individuals who are generally more inclined to stress and worry are also more likely to become cynical about the work situation. However, increases in anxiety symptoms have previously been linked to burnout-associated 
cynicism, with cynicism seen as the causal factor [32]. Until further prove one could perhaps surmise that the tendency to worry and stress predisposes to cynicism and that cynicism fosters the incidence of anxiety.

The next step was to test for correlations between scores on the cynicism subscale and health risk indicators, including SF-36v2 summary scores on physical health, allostatic load, CRP and HRV. The summary scores on physical health declined with increases in cynicism, and this decline in health with increases in cynicism was supported by results of the majority of physiological health risk indicators used in the study. Allostatic load is said to reflect the cumulative cost of stress over time, i.e., the physiological wear and tear [33]. Positive associations between allostatic load and cynicism approached statistical significance $(r=0.360, \mathrm{p} 0.065)$ and thus hinted at a trend for individuals who are cynical about their work situation to have higher physiological wear and tear. A positive association was further seen with body mass index (BMI), said to be a reflection of metabolism and adipose tissue deposition. Of interest are the associations seen between CRP levels and cynicism. CRP is an acute phase protein, often used as inflammatory biomarker. Elevated CRP levels have been described with stress and life style associated diseases, while low CRP levels is seen as a marker of wellness $[24,34]$. The statistical significant positive correlations found in the present study between CRP levels and cynicism thus indicate a decline in health with increases in cynicism.

HRV offers a window into autonomic nervous system control of the heart and a decline in HRV is known to occur with chronic stress, as well as in metabolic, immune and cardiovascular disorders $[25,35,36,37])$. Statistical significant negative correlations were found in the present study between cynicism and HRV indicators for supine and standing total HRV, as well as for supine and standing low frequency bands HRV, while negative correlations approaching significance were found between cynicism and vagal measures of HRV (HF). This decrease in HRV with increases in cynicism points towards a decline in physical and/or psychological health with an increase in the degree to which individuals felt cynical about, or disengaged themselves emotionally from the work situation. 
In summary it can be said that the results of this study confirm the assumption of an association between stress and disengagement from the work situation (score on MBI-GS cynicism subscale). Adverse effects of work-related cynicism on physical health were suggested by the negative associations with the summary scores on physical health and HRV, respectively, and by the positive associations with heart rate, allostatic load and the inflammatory marker CRP.

\section{Conclusions}

Cynicism, as reflected by the MBI-GS, increases with increases in stress levels and could contribute to the decline in the health reported for burnout. It would be of interest to know whether the associations found between a decline in health and cynicism towards the work environment is mediated through cynicism-related poor health behaviours.

\section{References}

1. Maslach C, Jackson SE, Leiter MP. Maslach Burnout Inventory. 3rd ed. Palo Alto, CA: Consulting Psychologist Press; 1996.

2. Shirom A, Melamed, S, Toker, S, Berliner S, Shapira I. Burnout and Health Review: Current Knowledge and Future Research Directions. In: Hodgkinson GP, Ford JK, editors. International Review of Industrial and Organizational Psychology, Volume 20. Chichester, UK: John Wiley \& Sons, Ltd; 2005. doi: 10.1002/0470029307.ch7.)

3. Sokka L, Huotilainen M, Leinikka M, Korpela J, Henelius A, Alain C, Müller K, Pakarinen S. Alterations in attention capture to auditory emotional stimuli in job burnout: an event-related potential study. Int J Psychophysiol. 2014;94(3):427-36. doi: 10.1016/j.jpsycho.2014.11.001.

4. Hallsten L, Voss M, Stark S, Josephson M. Job burnout and job wornout as risk factors for longterm sickness absence. Work. 2011;38(2):181-92. doi: 10.3233/WOR-2011-1120. 
5. Shirom A, \& Melamed, S. Does burnout affect physical health? A review of the evidence. In:

Antoniou ASG, Cooper CL, editors. Research companion to organizational health psychology.

Cheltenham, UK: Edward Elgar. 2005. p599-622.

6. Kristensen TS, Borritz, M, Villadsen E, Christensen KB. The Copenhagen Burnout Inventory: A new tool for the assessment of burnout. Work Stress. 2005;19:192-207.

7. Danhof-Pont MB, van Veen T, Zitman FG. Biomarkers in burnout: a systematic review. J Psychosom Res. 2011;70(6):505-524.

8. Fries E. Hypocortisolemic disorders. In: Hellhammer DH, Hellhammer J, editors. Stress. The brainbody connection.Key Issues in Mental Health. Basel: Karger; 2008. p. 60-77. 2008.

doi:10.1159/000119044)

9. Olsson EMG, Roth WT, Melin L. Psychophysiological characteristics of women suffering from stress-related fatigue. Stress Health. 2010;26(2):113-26.

10. De Vente W, van Amsterdam JGC, Olff M, Kamphuis JH, Emmelkamp PMG. Burnout Is associated with reduced parasympathetic activity and reduced HPA Axis responsiveness, predominantly in males. BioMed Res Int. 2015;2015:431725. doi:10.1155/2015/431725.

11. Lennartsson AK, Sjörs A, Währborg P, Ljung T, Jonsdottir IH. Burnout and hypocortisolism - a matter of severity? A study on ACTH and cortisol responses to acute psychosocial stress. Front Psychiatry 2015;6:8. doi:10.3389/fpsyt.2015.00008.

12.Tian R, Hou G,Li D, Yuan T-F, A Possible Change Process of Inflammatory Cytokines in the Prolonged Chronic Stress and Its Ultimate Implications for Health. The Scientific World Journal, vol. 2014, Article ID 780616, 8 pages, 2014. doi:10.1155/2014/780616.

13. Norheim KB, Jonsson G, Omdal R. Biological mechanisms of chronic fatigue. Rheumatology. 2011;50(6):1009-1018. doi:10.1093/rheumatology/keq454 . 
14. Edwards LD, Heyman AH, Swidan S. Hypocortisolism: An evidence-based review. Integrative Medicine 2011:10(4):26-33.

15. Lorusso L, Mikhaylova SV, Capelli E, Ferrari D, Ngonga GK, Ricevuti G. Immunological aspects of chronic fatigue syndrome. Autoimmun Rev. 2009;8(4):287-91. doi: 10.1016/j.autrev.2008.08.003.

16. Viljoen M, Panzer A. Sickness Behaviour: Causes and Effects. SA Fam Pract. 2003;45(9):15-18.

17. Bianchi R, Boffy C, Hingray, C, Truchot D, Laurent E. Comparative symptomatology of burnout and depression. J Health Psychol. 2013;18(6):782-787.

18. Romano GF, Tomassi S, Russell A, Mondelli V, Pariante CM. Fibromyalgia and chronic fatigue: The underlying biology and related theoretical issues. In: Balon R, Wise TN, editors. Clinical challenges in the biopsychosocial interface. Update on psychosomatics for the 21st century. Adv Psychosom Med. Basel: Karger; 2015. pp 61-77. doi:10.1159/000369085.

19. Schaufeli WB, Leiter MP, Maslach C. Burnout: 35 years of research and practice. Career Development International. Vol. 14 No. 3, 2009.pp. 204-220. Emerald Group Publishing Limited. 1362-0436. DOI 10.1108/13620430910966406

20. Van Zyl ES, van der Walt HS. Manual for the Experience of Work and Life Circumstances Questionnaire. Pretoria: HSRC. 1991.

21. Spielberger C, Gorsuch R, Lushene R, Vagg P, Jacobs G. State-trait anxiety inventory (form Y). Redwood City: CA: Mind Garden. 1983.

22. Maruish ME. User's manual for the SF-36v2 Health Survey. 3rd ed. Lincoln, RI: Quality Metric Incorporated. 2011.

23. Seeman TE, Singer BH, Rowe JW, Horwitz RI, McEwen BS. Price of adaptation - Allostatic load and its health consequences: MacArthur studies of successful aging. Arch Intern Med. 1997;157(19):2259-68. 
24. Kao PC, Shiesh SC, Wu TJ. Serum C-reactive protein as a marker for wellness assessment. Clin Lab Sci. 2006;36(2):163-9.

25. Task Force of the European Society of Cardiology the North American Society of Pacing Electrophysiology. Heart Rate Variability: Standards of measurement, physiological interpretation, and clinical use. Circulation. 1996;93:1043-1065. doi:10.1161/01.CIR.93.5.1043.

26. Tarvainen MP, Ranta-aho PO, Karjalainen PA. An advanced detrending method with application to HRV analysis. IEEE Trans Biomed Eng. 2002;49(2):172-5.

27. Schroeder KE, Narkiewicz K, Kato M, Pesek C, Phillips B, Davison D, Somers VK. Personality type and neural circulatory control. Hypertension. 2000;36:830-833. doi: 10.1161/01.HYP.36.5.830.

28. Tindle H, Davis E, Kuller L. Attitudes and cardiovascular disease. Maturitas. 2010;67(2):108-113. doi: 10.1016/j.maturitas.2010.04.020,S

29. Janicki-Deverts D, Cohen S, Doyle WJ. Cynical hostility and stimulated Th1 and Th2 cytokine production. Brain Behav Immun. 2010;24(1):58-63.

30. Neuvonen E, Rusanen M, Solomon A, Ngandu T, Laatikainen T, Soininen H, Kivipelto $M$, Tolppanen A-M. Late-life cynical distrust, risk of incident dementia and mortality in a populationbased cohort. Neurology. 2014;82:2205-12.

31. Why YP, Johnston DW. Cynicism, anger and cardiovascular reactivity during anger recall and human-computer interaction. Int J Psychophysiol. 2008;68(3):219-227.

32. Ding Y, Qu J, Yu X, Wang S. The mediating effects of burnout on the relationship between anxiety symptoms and occupational stress among community healthcare workers in China: a cross-sectional study. PLoS One. 2014;11;9(9):e107130. doi: 10.1371/journal.pone.0107130. eCollection 2014.

33. McEwen BS, Stellar E. Stress and the individual. Mechanisms leading to disease. Arch Intern Med. 1993;27;153(18):2093-101. 
34. Chandrashekara S. C - reactive protein: An inflammatory marker with specific role in physiology, pathology and diagnosis. IJRCI. 2014;2(S1):SR3.

35. Thayer JF, Åhs F, Fredrikson M, Sollers III JJ, Wager TD. A meta-analysis of heart rate variability and neuroimaging studies: Implications for heart rate variability as a marker of stress and health. Neurosci Biobehav Rev. 2012;36:747-756.

36. Hillebrand S, Gas KB, de Mutsert R, Swenne CA, Jukema JW, Middeldorp S, Rosendaal FR, Dekkers OM. Heart rate variability and first cardiovascular event in populations without known cardiovascular disease: meta-analysis and dose-response meta-regression. Europace 2013;15:742749. doi:10.1093/europace/eus341.

37. Wulsin LR, Horn PS, Perry JL, Massaro J, D'Agostino R. Autonomic imbalance as a predictor of metabolic risks, cardiovascular disease, diabetes, and mortality. J Clin Endocrinol Metab. 2015;100(6):2443-8. doi: 10.1210/jc.2015-1748. 